\title{
The different clinical faces of obstructive sleep apnoea: a cluster analysis
}

\author{
Lichuan Ye ${ }^{1}$, Grace W. Pien², Sarah J. Ratcliffe ${ }^{3}$, Erla Björnsdottir ${ }^{4,5}$, \\ Erna Sif Arnardottir 4,5 , Allan I. Pack ${ }^{6}$, Bryndis Benediktsdottir ${ }^{4,5}$ and \\ Thorarinn Gislason ${ }^{4,5}$
}

\begin{abstract}
Affiliations: 'Boston College William F. Connell School of Nursing, Chestnut Hill, MA, USA. ${ }^{2}$ Johns Hopkins University School of Medicine, Baltimore, MD, USA. ${ }^{3}$ Dept of Biostatistics and Epidemiology, University of Pennsylvania Perelman School of Medicine, Philadelphia, PA, USA. "Dept of Respiratory Medicine and Sleep, Landspitali, The National University Hospital of Iceland, Reykjavik, Iceland. ${ }^{5}$ Faculty of Medicine, School of Health Sciences, University of Iceland, Reykjavik, Iceland. ${ }^{6}$ Division of Sleep Medicine/Dept of Medicine, Center for Sleep and Circadian Neurobiology, University of Pennsylvania Perelman School of Medicine, Philadelphia, PA, USA.
\end{abstract}

Correspondence: Lichuan Ye, Boston College, William F. Connell School of Nursing, Cushing Hall 423, 140 Commonwealth Avenue, Chestnut Hill, MA 02467, USA. E-mail: yelabc.edu

ABSTRACT Although commonly observed in clinical practice, the heterogeneity of obstructive sleep apnoea (OSA) clinical presentation has not been formally characterised.

This study was the first to apply cluster analysis to identify subtypes of patients with OSA who experience distinct combinations of symptoms and comorbidities. An analysis of baseline data from the Icelandic Sleep Apnoea Cohort (822 patients with newly diagnosed moderate-to-severe OSA) was performed.

Three distinct clusters were identified. They were classified as the "disturbed sleep group" (cluster 1), "minimally symptomatic group" (cluster 2) and "excessive daytime sleepiness group" (cluster 3), consisting of $32.7 \%, 24.7 \%$ and $42.6 \%$ of the entire cohort, respectively. The probabilities of having comorbid hypertension and cardiovascular disease were highest in cluster 2 but lowest in cluster 3 . The clusters did not differ significantly in terms of sex, body mass index or apnoea-hypopnoea index.

Patients with OSA have different patterns of clinical presentation, which need to be communicated to both the lay public and the professional community with the goal of facilitating care-seeking and early identification of OSA. Identifying distinct clinical profiles of OSA creates a foundation for offering more personalised therapies in the future.

@ERSpublications

This study identified 3 different subtypes of patients with obstructive sleep apnoea based on clinical presentations http://ow.ly/AjuEZ

Received: Feb 172014 | Accepted after revision: July 122014 | First published online: Sept 032014

Support statement: This work was supported by the NIH grants HL072067 for "A Family Linkage Study of Obstructive Sleep Apnoea" and P01 HL094307 for "Endophenotypes of Sleep Apnoea and Role of Obesity", the Eimskip Fund of the University of Iceland and the Landspitali University Hospital Research Fund (Reykjavik, Iceland).

Conflict of interest: Disclosures can be found alongside the online version of this article at erj.ersjournals.com

Copyright @ERS 2014 


\section{Introduction}

Although commonly observed in clinical practice, the various clinical presentations of obstructive sleep apnoea (OSA) have not been formally characterised. Lack of knowledge of the heterogeneity of OSA clinical presentation may pose critical challenges to its clinical recognition, resulting in missed or delayed diagnosis. Unsupervised cluster analysis has recently been used to identify subtypes of patients who are diagnosed with a particular disorder, such as asthma, chronic obstructive pulmonary disease (COPD), fibromyalgia and Parkinson's disease [1-4]. With the goal of better understanding the heterogeneity of OSA clinical presentations, this study innovatively applied cluster analysis to identify subgroups of OSA patients who experience distinct combinations of symptoms and comorbidities. In addition, we examined whether patient subgroups differed with regard to patient demographics and other characteristics such as sex, age, body mass index (BMI), apnoea-hypopnoea index (AHI), and health status.

\section{Methods \\ Study subjects}

Subjects in our analysis are from the Icelandic Sleep Apnoea Cohort (ISAC), a clinic-based patient cohort from the entire population of Iceland. Detailed descriptions of the ISAC have been published previously [5-7]. The study inclusion criteria was patients with moderate-to-severe OSA (AHI $\geqslant 15$ events $\cdot$ hour $\left.^{-1}\right)$, referred for positive airway pressure (PAP) treatment to the Dept of Pulmonary Medicine and Sleep, the National University Hospital of Iceland, Reykjavik, the sole provider of PAP therapy in Iceland (total population 320000 ). In Iceland all patients with AHI $\geqslant 15$ events·hour ${ }^{-1}$ are referred for PAP therapy. Over $90 \%$ of eligible and approached subjects agreed to participate. Among the detailed assessments performed at baseline prior to starting PAP, each participant completed a type three sleep study, physical examination and a survey about sleep-related health issues. Our sample included 822 patients from the ISAC who had baseline data available. The study was approved by the Iceland National Bioethics Committee, the Data Protection Authority of Iceland, and the Institutional Review Board at the University of Pennsylvania (Philadelphia, PA, USA).

\section{Questionnaires}

Several standardised and previously validated instruments were embedded in the survey to evaluate sleeprelated symptoms and health status, including the Basic Nordic Sleep Questionnaire (BNSQ) [8], Epworth Sleepiness Scale (ESS) [9], and 12-item Short Form Health Survey (SF-12) [10]. Patients also answered questions about restless leg syndrome (RLS) symptoms [11] and their medical history such as physiciandiagnosed hypertension and diabetes.

The BNSQ is a scale assessing the frequency of subjective sleep complaints in the past 3 months, ranging from 1 (never or less than once per month), 2 (less than once a week), 3 (once or twice a week), 4 (three to five times a week), to 5 (every night or almost daily) [8]. The BNSQ is frequently used in epidemiological and genetic research for OSA with demonstrated validity [12] and has been used widely in routine clinical practice in the Nordic countries [8].

\section{Choosing symptom and comorbidity variables for cluster analysis}

We included a total of 23 variables in the cluster analysis to represent clinically significant and prevalent symptoms and comorbidities in the OSA population (a full list of variables is outlined in the results section).

The majority of symptom variables were dichotomised variables derived from responses to the questions on the BNSQ. In general, we defined a specific symptom as "present" if it occurred at least once or twice per week (response score: $3-5$ ). To capture the existence of clinically significant snoring and whether the snoring disturbed the spouse's sleep, we coded the snoring variable as 0 (doesn't snore loudly), 1 (snores loudly but doesn't disturb their spouse's sleep), and 2 (snores loudly and disturbs their spouse's sleep). Given our clinical observation that RLS is common among patients with untreated OSA, we included the presence of RLS in the cluster analysis using the same definition we adopted in a previous epidemiological study [13]. In addition, we included the ESS score as a continuous variable to indicate the overall degree of daytime sleepiness.

The presence of four comorbid conditions was included in the analysis: hypertension, diabetes, cardiovascular disease and obstructive lung disease. These conditions were most commonly reported on the survey and were, thus, included in the cluster analysis. The diagnosis of each comorbidity was validated against medical records. Hypertension and diabetes were defined as a physician diagnosis combined with treatment with appropriate medication. Cardiovascular disease was defined as a physician diagnosis of coronary artery occlusion (myocardial infarction or heart attack), heart failure and/or stroke. Obstructive lung disease was defined as a physician diagnosis of COPD, emphysema and/or asthma. 


\section{Statistical analysis}

Latent class analysis (LCA) [14, 15] was used to cluster subjects into groups based on symptoms and presence of comorbidities. The basic principle of cluster analysis is that individuals are grouped together based on specified variables so that members of each cluster are as similar as possible to others within the cluster, but as different as possible compared with those in other clusters. Two tests of model fit, the likelihood ratio test and Bayesian information criterion, were used to determine the optimal number of clusters. Conditional probabilities or the cluster-specific probabilities were calculated, indicating the probability of a specific symptom or comorbid condition being present in the cluster. Probabilities between clusters were compared via odds ratios, with unadjusted $\mathrm{p} \leqslant 0.002$ being considered statistically significant after a Holm's adjustment for multiple comparisons. LCA was performed using Mplus 5.1 (www.statmodel.com) [16].

After the clusters or patient subgroups were identified, differences among clusters with regard to patient demographic and other characteristics, including sex, age, BMI, AHI, oxygen desaturation index (ODI), minimum oxygen saturation during the sleep study and health status (SF-12 scores), were examined via Chi-squared, ANOVA, or Kruskal-Wallis equality-of-populations rank tests, as appropriate. Analyses were conducted using StataMP (Stata Statistical Software, Release 12; StataCorp, College Station, TX, USA) and p-values $<0.05$ were considered statistically significant.

\section{Results}

\section{Sample characteristics}

The total cohort sample characteristics are summarised in table 1. The sample consisted predominantly of middle-aged obese males with severe OSA. The frequencies of each symptom and comorbid condition for the entire cohort are detailed in table 2.

\section{Three distinct clusters}

Three distinct clusters were identified based on symptom experiences and the existence of major comorbidities. Table 2 lists the conditional probabilities of each symptom and comorbid condition present in each cluster, which are compared between clusters in table 3. To highlight the major differences between clusters, figure 1 presents the probability of having certain symptoms within each cluster in a comparable manner.

Cluster 1 was the "disturbed sleep group". Members of cluster 1 (32.7\% of the entire cohort) had the highest probability of experiencing insomnia-related symptoms, including difficulty falling asleep at night (44.3\%), waking up too early and difficulty falling back to sleep (60.8\%), and most prominently, waking up often during the night $(90.3 \%)$. Other nocturnal symptoms were also prominent, such as heavy perspiration (61.7\%), being restless (74.8\%), RLS symptoms (42.6\%), and sudden awakening due to gasping for breath (21.1\%).

Cluster 2 was the "minimally symptomatic group," consisting of $24.7 \%$ of the entire cohort. For the majority of symptoms, the probability was markedly lower in cluster 2 than in the other two clusters. Members of cluster 2 were much more likely to feel rested upon waking up (78.3\%) compared with those in cluster $1(38.7 \%)$ or in cluster $3(24.3 \%)$.

Cluster 3 (42.6\% of the cohort) was the "excessive daytime sleepiness group," with a significantly higher ESS score $(15.7 \pm 0.6)$ than cluster $1(9.5 \pm 0.7)$ and cluster $2(7.9 \pm 0.6)$, and a markedly higher probability

\section{TABLE 1 Demographic and clinical characteristics of the total cohort and by clusters}

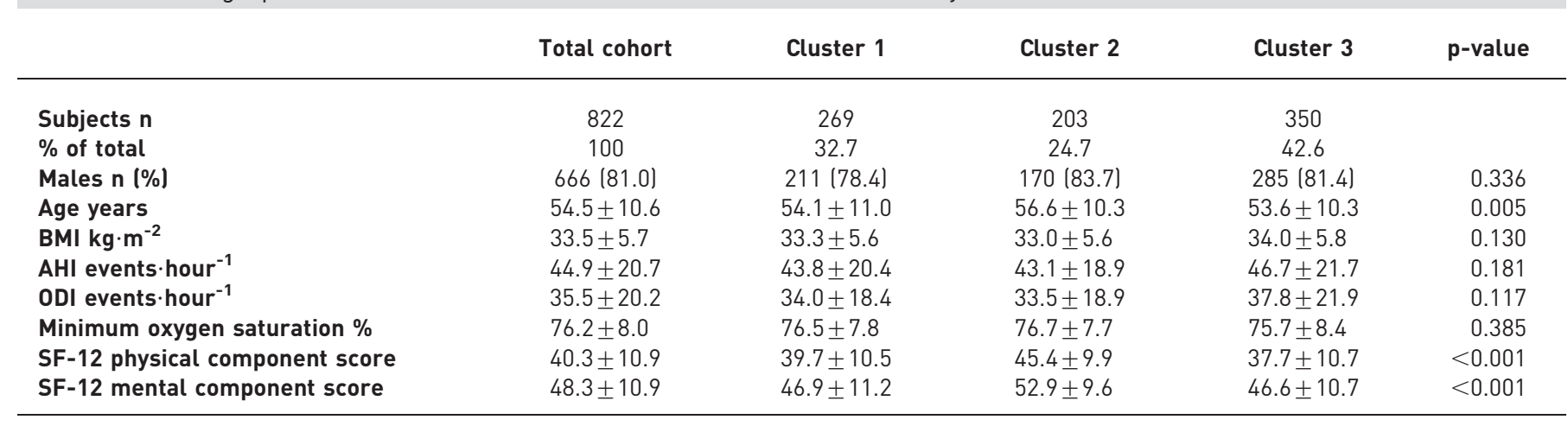

Data are presented as mean \pm SD, unless otherwise stated. BMI: body mass index; AHI: apnoea-hypopnoea index; ODI: oxygen desaturation index; SF-12: 12-item Short Form Health Survey. 
of complaining of sleepiness-related symptoms, such as falling asleep involuntarily during the day $(64.6 \%$ versus $11.1 \%$ in cluster 1 and $8.6 \%$ in cluster 2 ), and dozing off when driving (38.2\% versus $3.4 \%$ in cluster 1 and $4.4 \%$ in cluster 2). They also had a somewhat higher likelihood of presenting with classic OSA symptoms, such as night-time breathing pauses and loud snoring disturbing their spouse's sleep.

Among the three clusters, the probabilities of having comorbid hypertension, diabetes and cardiovascular disease were highest in cluster 2 but lowest in cluster 3. For example, cluster 2 was more likely to have comorbid hypertension $(49.6 \%$ versus $41.6 \%, \mathrm{OR}=1.38 ; \mathrm{p}<0.001)$ and cardiovascular disease $(18.3 \%$ versus $11.9 \%, \mathrm{OR}=1.67 ; \mathrm{p}=0.001)$ when compared with cluster 3.

We ran two additional analyses to ensure the stability of the three clusters in this sample. First, we repeated the cluster analysis by defining symptoms as "present" if they occurred at least three to five times a week. Although the selection of this higher threshold led to an overall lower prevalence of symptoms, a similar set of three distinct clusters were identified based on the combination of symptoms and comorbidities. Secondly, we included age, sex and AHI in the cluster analysis in addition to the original 23 variables. Similar results were identified with the additional three variables in the model.

\section{Differences among the three clusters}

As indicated in table 1, no statistical difference was observed in sex, BMI, AHI, ODI or the minimum oxygen saturation among the three clusters. Although mean age was statistically different among clusters, this difference ( 54.1 versus 56.6 versus 53.6 years) was unlikely to be clinically meaningful. Both the SF-12 physical and mental component scores significantly differed among clusters $(\mathrm{p}<0.001)$, even after adjusting for sex, age and BMI, with the highest scores indicating the best physical and mental health status being reported by members of cluster 2 . In addition, we examined the use of hypnotics and found that the frequency of hypnotic use was significantly higher in members of cluster $1(16.4 \%$; $=0.017)$ than those of cluster $2(8.9 \%)$ and cluster $3(10.1 \%)$.

TABLE 2 Symptom experiences and comorbidities of subjects in the total cohort and by clusters

\section{Total cohort Cluster 1}

Cluster 2

Cluster 3

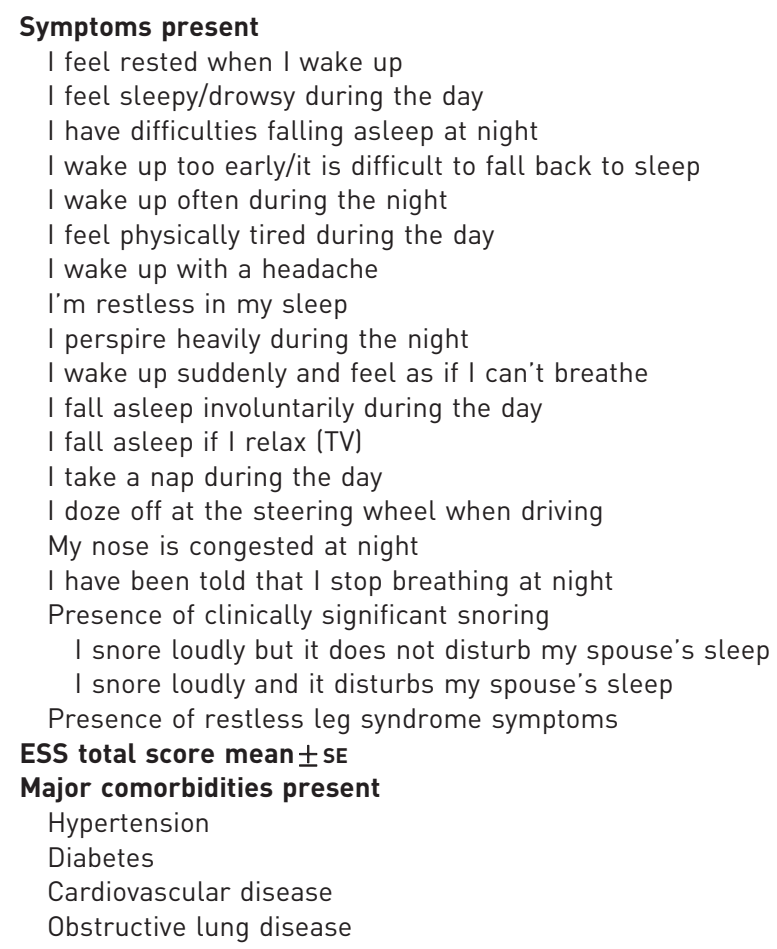

\begin{tabular}{|c|c|c|c|}
\hline 42.6 & 38.7 & 78.3 & 24.3 \\
\hline 87.8 & 90.9 & 68.3 & 97.1 \\
\hline 28.9 & 44.3 & 13.6 & 25.8 \\
\hline 44.9 & 60.8 & 26.8 & 43.3 \\
\hline 73.0 & 90.3 & 43.5 & 77.0 \\
\hline 87.8 & 96.3 & 59.6 & 98.1 \\
\hline 23.7 & 24.6 & 6.4 & 33.2 \\
\hline 60.4 & 74.8 & 24.9 & 70.5 \\
\hline 49.5 & 61.7 & 24.7 & 54.8 \\
\hline 18.0 & 21.1 & 1.8 & 25.3 \\
\hline 32.8 & 11.1 & 8.6 & 64.6 \\
\hline 77.5 & 60.1 & 65.0 & 98.8 \\
\hline 45.4 & 49.4 & 25.7 & 54.0 \\
\hline 18.1 & 3.4 & 4.4 & 38.2 \\
\hline 48.4 & 54.4 & 27.5 & 56.3 \\
\hline 77.1 & 75.0 & 67.5 & 84.6 \\
\hline 9.9 & 13.6 & 10.3 & 6.9 \\
\hline 81.9 & 79.2 & 71.7 & 89.8 \\
\hline 36.8 & 42.6 & 14.5 & 45.6 \\
\hline $11.7 \pm 0.2$ & $9.5 \pm 0.7$ & $7.9 \pm 0.6$ & $15.7 \pm 0.6$ \\
\hline 45.7 & 47.8 & 49.6 & 41.6 \\
\hline 8.7 & 8.8 & 10.5 & 7.5 \\
\hline 14.4 & 14.6 & 18.3 & 11.9 \\
\hline 18.7 & 21.3 & 16.2 & 18.2 \\
\hline
\end{tabular}

Data are presented as \%, unless otherwise stated. ESS: Epworth Sleepiness Scale. 
TABLE 3 Odds ratios for comparisons of probabilities between clusters

\begin{tabular}{|c|c|c|c|c|c|c|}
\hline & \multicolumn{2}{|c|}{$\begin{array}{c}\text { Cluster } 1 \text { versus } \\
\text { cluster } 2\end{array}$} & \multicolumn{2}{|c|}{$\begin{array}{c}\text { Cluster } 1 \text { versus } \\
\text { cluster } 3\end{array}$} & \multicolumn{2}{|c|}{$\begin{array}{c}\text { Cluster } 2 \text { versus } \\
\text { cluster } 3\end{array}$} \\
\hline & OR & p-value & OR & p-value & OR & p-value \\
\hline \multicolumn{7}{|l|}{ Symptoms present } \\
\hline I feel rested when I wake up & 0.18 & 0.001 & 1.97 & 0.001 & 11.24 & 0.003 \\
\hline I feel sleepy/drowsy during the day & 4.65 & 0.092 & 0.30 & 0.130 & 0.07 & 0.029 \\
\hline I wake up often during the night & 12.06 & 0.016 & 2.76 & 0.038 & 0.23 & 0.001 \\
\hline I feel physically tired during the day & 17.68 & 0.202 & 0.51 & 0.357 & 0.03 & 0.094 \\
\hline I wake up with a headache & 4.75 & 0.024 & 0.66 & 0.002 & 0.14 & 0.006 \\
\hline I'm restless in my sleep & 8.97 & 0.004 & 1.24 & 0.006 & 0.14 & 0.003 \\
\hline I perspire heavily during the night & 4.90 & 0.001 & 1.33 & $<0.001$ & 0.27 & 0.001 \\
\hline I wake up suddenly and feel as if I can't breathe & 14.63 & 0.649 & 0.79 & 0.002 & 0.05 & 0.651 \\
\hline I have been told that I stop breathing at night & 1.44 & 0.001 & 0.55 & 0.001 & 0.38 & $<0.001$ \\
\hline \multicolumn{7}{|l|}{ Clinically significant snoring } \\
\hline I snore loudly but it does not disturb my spouse's sleep & 2.83 & 0.034 & 0.44 & 0.091 & 0.15 & 0.028 \\
\hline I snore loudly and it disturbs my spouse's sleep & 1.51 & 0.008 & 0.43 & 0.007 & 0.29 & 0.001 \\
\hline Restless leg syndrome symptoms & 4.37 & 0.003 & 0.89 & 0.001 & 0.20 & 0.001 \\
\hline \multicolumn{7}{|l|}{ Major comorbidities present } \\
\hline Hypertension & 0.93 & 0.002 & 1.29 & 0.001 & 1.38 & $<0.001$ \\
\hline Diabetes & 0.83 & 0.083 & 1.20 & 0.079 & 1.44 & 0.023 \\
\hline Cardiovascular disease & 0.76 & 0.012 & 1.27 & 0.007 & 1.67 & 0.001 \\
\hline Obstructive lung disease & 1.40 & 0.007 & 1.21 & 0.001 & 0.87 & 0.002 \\
\hline
\end{tabular}

Bold font indicates variables with statistically significant differences between clusters/groups ( $\leqslant \leqslant 0.002$ ).

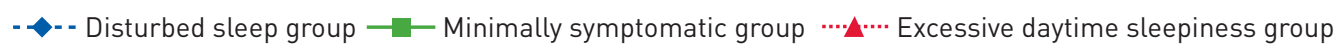

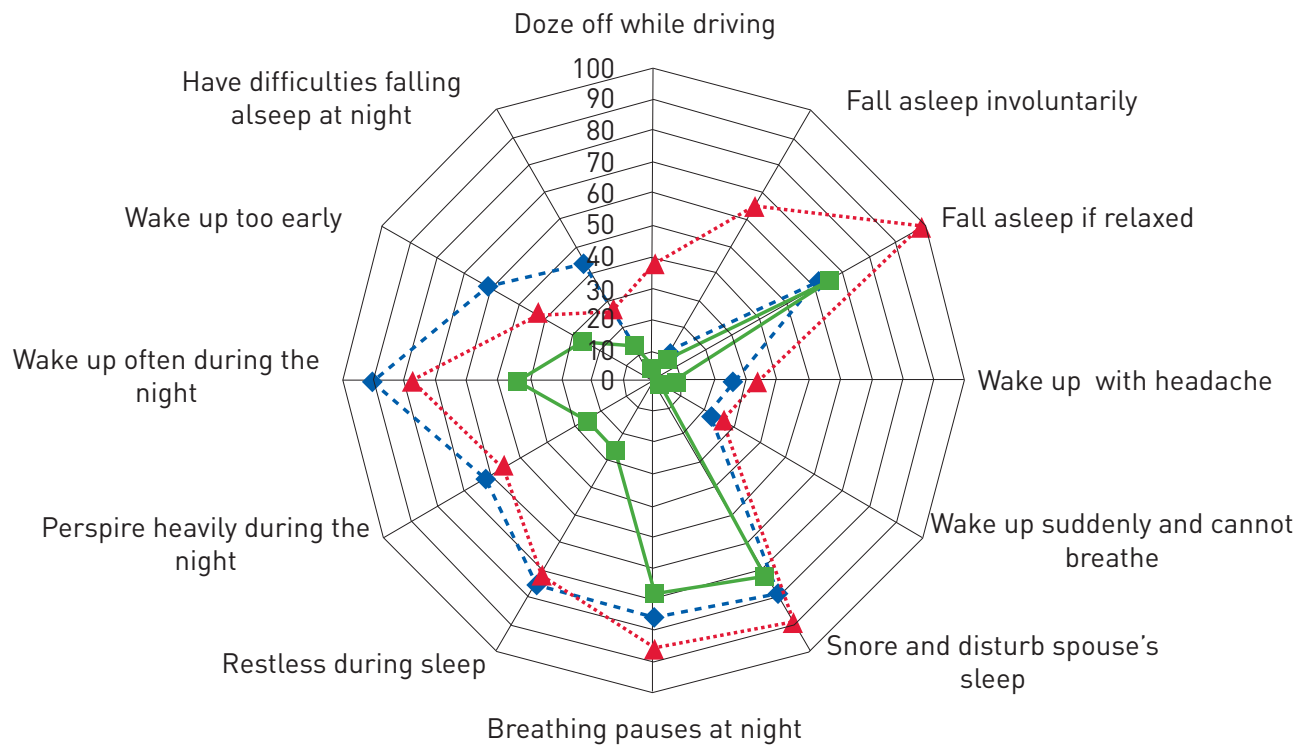

FIGURE 1 Probability of having a symptom within each cluster. The conditional probabilities of 12 symptoms (selected from the complete list in Table 2) are shown to highlight the major differences among clusters. 


\section{Discussion}

To the best of our knowledge, this is the first attempt to explore the heterogeneity of OSA clinical presentations using a data-driven approach. Our results suggest that there are different clinical subtypes of OSA. The major strengths of this study are innovative data analysis and the large clinical cohort representing patients with OSA who need PAP treatment across the entire population of Iceland. Furthermore, the inclusion of a comprehensive number of symptoms enhances our understanding of the OSA symptom experience.

As a novel approach with the potential to refine phenotypic diagnostic criteria [17], cluster analysis has been used in other medical conditions to identify symptom patterns and clinical phenotypes [1-4]. A recent study using cluster analysis identified five distinct clinical phenotypes of asthma, illustrating heterogeneity in the clinical presentation of asthma and clinically relevant differences in treatment outcomes among various phenotypes [3]. Another example of this approach is illustrated by an analysis of the presenting symptoms for myocardial infarction: five clusters or patient subgroups with distinct symptom patterns were identified, with none of these clusters including all of the symptoms that are considered typical of myocardial infarction [18].

Understanding differences in patterns of OSA clinical presentation is especially important. Patients in cluster 1 and cluster 2, which together made up over half of the sample, were less likely to present with clinically significant daytime sleepiness (i.e. mean ESS score $<10$ ). Although the majority of the individuals in cluster 1 and cluster 2 reported other classic OSA symptoms such as breathing pauses at night and loud snoring that disturbed their spouses' sleep, they were less likely to do so than patients in cluster 3 . As supported by the seminal Wisconsin Sleep Cohort Study [19], more individuals with OSA in the community who may be less symptomatic have not yet been referred and diagnosed. Therefore, current clinical practice and research, which emphasise only a few "typical" symptoms such as snoring and daytime sleepiness, may have created a potentially problematic image of a stereotypical OSA patient.

Although OSA severity is widely classified using the AHI [20], this objective index does not consider the marked heterogeneity of differing clinical presentations of OSA. Remarkably, as demonstrated by our observation, in three patients with essentially identical AHIs, one can be a loud snorer with excessive daytime sleepiness, another may mainly complain of disturbed sleep, while the third may not be bothered by any major OSA-related symptoms but is more likely to have coexisting hypertension or cardiovascular disease. Our findings suggest that identifying clinical presentations based on combinations of symptoms and comorbidities can more fully capture the spectrum of the OSA experience than relying solely on indices such as AHI or ODI.

Members of cluster 1 are distinguished by having the most disturbed sleep, particularly showing insomnia symptoms. A relatively high prevalence of other symptoms, such as RLS, nocturnal sweating [5] and frequent awakening due to gasping for breath, may also have contributed to their insomnia complaints. Although numerous studies, including studies using an insomnia severity scale to quantify insomnia, have documented the high prevalence of insomnia symptoms in patients with OSA [21-27], the evaluation of insomnia has not been recommended as part of routine screening for OSA [28]. A recent study in family medicine patients found insomnia to be a significant predictor of a clinical diagnosis of OSA, independent of BMI and excessive daytime sleepiness [29]. The relatively high likelihood of hypnotic use in these patients further emphasises the need to develop strategies for adequately managing comorbid insomnia.

Unlike typical patients with insomnia disorder [30], members of cluster 1 had a higher probability of daytime napping. The disturbed sleep may have exacerbated daytime sleepiness in these untreated OSA patients [22]. Meanwhile, untreated OSA could worsen insomnia in these patients. It is likely that repeated breathing disturbances in untreated OSA results in sleep fragmentation and hence difficulties maintaining sleep. Repeated arousals that result from abnormal respiratory events may also further aggravate the hyperarousal associated with typical insomnia. Insomnia symptoms in a group of OSA patients have been reported to significantly improve after 24 months of PAP treatment [26]. By contrast, a recent study conducted by our team found that PAP treatment only significantly reduced symptoms of difficulties maintaining sleep [31]. We found that other subtypes of insomnia, including difficulties initiating sleep and early morning awakenings, tended to persist regardless of PAP treatment and that difficulty falling asleep even negatively impacted PAP adherence [31]. These results highlight the importance of assessing insomnia subtypes in the management of OSA. Specific treatment for insomnia prior to or combined with PAP treatment, such as cognitive behavioural therapy, could be beneficial, at least for some OSA patients affected by difficulty falling asleep.

We found that members of cluster 2, the minimally symptomatic group, reported the most favourable physical and mental health status. This finding supports symptom status as a key predictor of quality of life, 
and the importance of comprehensive evaluation of symptoms for improving quality of life in patients with OSA [32]. In contrast to the finding that excessive daytime sleepiness was associated with an increased risk of future cardiovascular disease in community-dwelling elderly individuals [33], members of cluster 3, the excessive daytime sleepiness group, had a lower probability of having comorbid hypertension, diabetes and cardiovascular disease than members of other clusters. The lag time between initial OSA symptoms and OSA diagnosis can vary widely, with the average duration reported to be approximately 10 years [34]. Members of cluster 3, who were sleepier and more likely to report classic features of OSA, may have had a shorter lag time. By contrast, members of cluster 2 who were minimally symptomatic may have had a longer lag time, leading to a longer duration of exposure to untreated OSA and, thus, a higher probability of developing comorbidities. Nevertheless, they were neither more obese nor significantly older, and they did not have higher AHIs, than other members of the cohort. In addition to lag time prior to OSA diagnosis, other factors that were not examined in this study, such as the existence of a bed partner, may have a significant impact on the clinical presentations of OSA and deserve further investigation.

Our study focuses on one of the goals of personalised medicine: to identify distinct subtypes among individuals with a particular disease with the goal of more precisely targeted therapies. As such, our study population is not intended to be a community-based cohort. Thus, we recruited 822 consecutively diagnosed patients with OSA in Iceland. There have been extensive efforts in Iceland to educate primary care and other physicians about OSA, resulting in a high level of awareness of this disorder. All patients with identified OSA are referred to a single location for treatment. The high participation rate for research in Iceland is another substantial advantage. Thus, the ISAC cohort is representative of all patients recently diagnosed with OSA in one country, minimising referral bias.

Nevertheless, one limitation of this study is that data were collected exclusively from persons with moderate-to-severe OSA. Our subjects were predominately middle-aged obese males, who are typical of patients with more severe OSA. Although we have conducted additional analyses to ensure the stability of the clusters, further validation in independent cohorts including a wider variety of demographics and levels of OSA severity is needed. Although the overall patterns of clinical presentations of OSA were not significantly influenced by patient age, sex and AHI in our sample, these important sociodemographic variables may account for different clinical presentations of OSA [35] and should be carefully considered in future investigations with more diverse samples. Some symptoms and/or comorbidities that may be common in the OSA population, such as depression and cognitive decline, were not included in this study. Future investigations need to consider more fully the existence of comorbid conditions. Finally, although we did not observe differences among clusters in AHI, AHI may have been underestimated among individuals with insomnia symptoms, given the inability to precisely measure total sleep time using type 3 portable monitors.

In conclusion, our findings are clinically significant in two major aspects. First, identification of subtypes of OSA improves our knowledge and awareness of the heterogeneity of OSA clinical presentation. Communication of this knowledge to both the lay public and the professional community can facilitate care-seeking and early identification of OSA. This is particularly important for individuals with "atypical" OSA symptom experiences and those who are less symptomatic. Although debate persists regarding the need for treatment of "asymptomatic" OSA, a number of studies have demonstrated that minimally symptomatic individuals with OSA are still at increased cardiovascular risk [36-38]. Secondly, identifying distinct clinical profiles of OSA creates a foundation for offering more personalised therapies in the future. Follow-up studies are needed to examine whether the response to treatment differs among OSA patients with distinct patterns of clinical presentations, particularly in terms of changes in symptom presentation, blood pressure and cardiovascular comorbidities. Further investigation is also needed to explore the underlying mechanisms of distinct clinical phenotypes, including the genotypic differences associated with different clinical presentations.

\section{Acknowledgements}

The authors are grateful to Sigrun Gudmundsdottir, Lovisa Gudmundsdottir, Magdalena Osk Sigurgunnarsdottir and Kristján Andri Kristjánsson at the Sleep Centers of Landspitali University Hospital, Rekjavik, Iceland, and to Matthew Thorne-Fitzgerald, Robert Hachadoorian and the other staff at the University of Pennsylvania, Philadelphia, PA, USA, who helped assemble and analyse the data.

\section{References}

Docampo E, Collado A, Escaramis G, et al. Cluster analysis of clinical data identifies fibromyalgia subgroups. PLoS One 2013; 8: e74873.

2 Erro R, Vitale C, Amboni M, et al. The heterogeneity of early Parkinson's disease: a cluster analysis on newly diagnosed untreated patients. PLoS One 2013; 8: e70244.

3 Moore WC, Meyers DA, Wenzel SE, et al. Identification of asthma phenotypes using cluster analysis in the Severe Asthma Research Program. Am J Respir Crit Care Med 2010; 181: 315-323. 
Garcia-Aymerich J, Gómez FP, Benet M, et al. Identification and prospective validation of clinically relevant chronic obstructive pulmonary disease (COPD) subtypes. Thorax 2011; 66: 430-437.

5 Arnardottir ES, Janson C, Bjornsdottir E, et al. Nocturnal sweating - a common symptom of obstructive sleep apnoea: the Icelandic sleep apnoea cohort. BMJ Open 2013; 3: e002795.

6 Arnardottir ES, Maislin G, Jackson N, et al. The role of obesity, different fat compartments and sleep apnea severity in circulating leptin levels: the Icelandic Sleep Apnea Cohort study. Int J Obes (Lond) 2013; 37: 835-842.

7 Arnardottir ES, Maislin G, Schwab RJ, et al. The interaction of obstructive sleep apnea and obesity on the inflammatory markers C-reactive protein and interleukin-6: the Icelandic Sleep Apnea Cohort. Sleep 2012; 35: 921-932.

8 Partinen M, Gislason T. Basic Nordic Sleep Questionnaire (BNSQ): a quantitated measure of subjective sleep complaints. J Sleep Res 1995; 4: 150-155.

9 Johns MW. A new method for measuring daytime sleepiness: the Epworth sleepiness scale. Sleep 1991; 14: 540-545.

10 Ware J Jr, Kosinski M, Keller SD. A 12-Item Short-Form Health Survey: construction of scales and preliminary tests of reliability and validity. Med Care 1996; 34: 220-233.

11 Allen RP, Picchietti D, Hening WA, et al. Restless legs syndrome: diagnostic criteria, special considerations, and epidemiology. A report from the restless legs syndrome diagnosis and epidemiology workshop at the National Institutes of Health. Sleep Med 2003; 4: 101-119.

12 Fedson AC, Pack AI, Gislason T. Frequently used sleep questionnaires in epidemiological and genetic research for obstructive sleep apnea: a review. Sleep Med Rev 2012; 16: 529-537.

13 Benediktsdottir B, Janson C, Lindberg E, et al. Prevalence of restless legs syndrome among adults in Iceland and Sweden: lung function, comorbidity, ferritin, biomarkers and quality of life. Sleep Med 2010; 11: 1043-1048.

14 McLachlan GJ, Peel D. Finite Mixture Models. New York, John Wiley \& Sons, Inc., 2000.

15 Vermunt JK, Magidson J. Latent class cluster analysis. In: Hagenaars JA, McCutcheon AL, eds. Applied Latent Class Analysis. Cambridge, Cambridge University Press, 2002; pp. 89-106.

16 Muthén LK, Muthén BO. Mplus User's Guide. 5th Edn. Los Angeles, Muthén \& Muthén, 2007.

17 Miaskowski C, Aouizerat BE, Dodd M, et al. Conceptual issues in symptom clusters research and their implications for quality-of-life assessment in patients with cancer. J Natl Cancer Inst Monogr 2007; 39-46.

18 Ryan CJ, DeVon HA, Horne R, et al. Symptom clusters in acute myocardial infarction: a secondary data analysis. Nurs Res 2007; 56: 72-81.

19 Young T, Palta M, Dempsey J, et al. The occurrence of sleep-disordered breathing among middle-aged adults. N Engl J Med 1993; 328: 1230-1235.

20 Sleep-related breathing disorders in adults: recommendations for syndrome definition and measurement techniques in clinical research. The Report of an American Academy of Sleep Medicine Task Force. Sleep 1999; 22: $667-689$.

21 Luyster FS, Buysse DJ, Strollo PJ Jr. Comorbid insomnia and obstructive sleep apnea: challenges for clinical practice and research. J Clin Sleep Med 2010; 6: 196-204.

22 Björnsdóttir E, Janson C, Gíslason T, et al. Insomnia in untreated sleep apnea patients compared to controls. J Sleep Res 2012; 21: 131-138.

23 Al-Jawder SE, Bahammam AS. Comorbid insomnia in sleep-related breathing disorders: an under-recognized association. Sleep Breath 2012; 16: 295-304.

24 Krell SB, Kapur VK. Insomnia complaints in patients evaluated for obstructive sleep apnea. Sleep Breath 2005; 9: $104-110$.

25 Smith S, Sullivan K, Hopkins W, et al. Frequency of insomnia report in patients with obstructive sleep apnoea hypopnea syndrome (OSAHS). Sleep Med 2004; 5: 449-456.

26 Nguyên XL, Rakotonanahary D, Chaskalovic J, et al. Insomnia related to sleep apnoea: effect of long-term autoadjusting positive airway pressure treatment. Eur Respir J 2013; 41: 593-600.

27 Nguyên XL, Chaskalovic J, Rakotonanahary D, et al. Insomnia symptoms and CPAP compliance in OSAS patients: a descriptive study using data mining methods. Sleep Med 2010; 11: 777-784.

28 Epstein LJ, Kristo D, Strollo PJ Jr, et al. Clinical guideline for the evaluation, management and long-term care of obstructive sleep apnea in adults. J Clin Sleep Med 2009; 5: 263-276.

29 Glidewell RN, Roby EK, Orr WC. Is insomnia an independent predictor of obstructive sleep apnea? J Am Board Fam Med 2012; 25: 104-110.

30 Bonnet MH, Arand DL. Hyperarousal and insomnia: state of the science. Sleep Med Rev 2010; 14: 9-15.

31 Björnsdóttir E, Janson C, Sigurdsson JF, et al. Symptoms of insomnia among patients with obstructive sleep apnea before and after two years of positive airway pressure treatment. Sleep 2013; 36: 1901-1909.

32 Ye L, Liang ZA, Weaver TE. Predictors of health-related quality of life in patients with obstructive sleep apnoea. J Adv Nurs 2008; 63: 54-63.

33 Empana JP, Dauvilliers Y, Dartigues JF, et al. Excessive daytime sleepiness is an independent risk indicator for cardiovascular mortality in community-dwelling elderly: the three city study. Stroke 2009; 40: 1219-1224.

34 Guilleminault C, Stoohs R, Kim YD, et al. Upper airway sleep-disordered breathing in women. Ann Intern Med 1995; 122: 493-501.

35 Morrell MJ, Finn L, McMillan A, et al. The impact of ageing and sex on the association between sleepiness and sleep disordered breathing. Eur Respir J 2012; 40: 386-393.

36 Kohler M, Craig S, Pepperell JC, et al. CPAP improves endothelial function in patients with minimally symptomatic OSA: results from a subset study of the MOSAIC trial. Chest 2013; 144: 896-902.

37 Ayers L, Ferry B, Craig S, et al. Circulating cell-derived microparticles in patients with minimally symptomatic obstructive sleep apnoea. Eur Respir J 2009; 33: 574-580.

38 Kohler M, Craig S, Nicoll D, et al. Endothelial function and arterial stiffness in minimally symptomatic obstructive sleep apnea. Am J Respir Crit Care Med 2008; 178: 984-988. 